Materials and Methods This open-label, multicenter phase 1a/ 1b clinical trial (NCT04096638) aims to enroll approximately 110 patients in the dose escalation (Part 1) and expansion cohorts (Part 2). Part 1 of the trial is a dose escalation study with IV SB11285 monotherapy followed by combination with the checkpoint inhibitor nivolumab. Part 1 Dose Escalation of the study will evaluate ascending doses of intravenously administered SB 11285 with respect to dose-limiting toxicities (DLTs), maximum tolerated dose (MTD), recommended phase 2 dose (RP2D) and the pharmacokinetic (PK)/pharmacodynamic profile as monotherapy and in combination with nivolumab. SB 11285, with a starting dose of $0.3 \mu \mathrm{g} / \mathrm{kg}$, will be administered as monotherapy weekly on Days 1, 8, 15, and 22 of repeated 28-day cycles in escalating doses and in combination with nivolumab administered on Q4W schedule. Part 2 Expansion Cohorts of the study will explore initial signs of efficacy in prespecified tumor types (such as Melanoma, HNSCC) using the recommended phase 2 dose (RP2D) of SB 11285 in combination with nivolumab. In addition, the biological effects of SB 11285 will be evaluated by changes in immune cell types and activation state, serum cytokines, and gene expression patterns indicative of activation of the immune compartment. The trial is being conducted at multiple sites in the U.S.

Disclosure Information A. Abbas: A. Employment (full or part-time); Modest; Spring Bank Pharmaceutical Inc. J. Strauss: E. Ownership Interest (stock, stock options, patent or other intellectual property); Modest; Abbvie, Abbott Laboratories, Bristol-Myers Squibb, Intuitive Surgical, Johnson \& Johnson, Merck. F. Consultant/Advisory Board; Modest; Tempus. Other; Modest; Dialectic Therapeutics. F. Janku: None. R. Karim: None. A. Olszanski: F. Consultant/Advisory Board; Modest; Bristol Myers Squibb. J.J. Luke: B. Research Grant (principal investigator, collaborator or consultant and pending grants as well as grants already received); Modest; All to institution for clinical trials unless noted) Abbvie, Bristol Myers Squibb, Medimmune, Necktar, Novartis, Merck, Leap, Incyte, Immunocore, Compugen, Corvus, Evil, Five Prime, Genentech, Immatic. F. Consultant/Advisory Board; Modest; Consultant: Akrevia, Algios, Array, Astellas,AstraZeneca, Bayer, Bristol Myers Squibb/Advisory Board:7 Hills, Actym, Alphamab Oncology, Mavu (now part of Abbvie), Pyxis, Spring Bank Pharma, Tempest. Other; Modest; Travel: Akrevia, Bayer, Bristol Myers Squibb, Reflexion, EMD Serono, Incyte, Janssen, Merck, Mersana, Novartis. K. Leach: A. Employment (full or part-time); Modest; Spring Bank Pharmaceuticals Inc. R. Iyer: A. Employment (full or part-time); Modest; Spring Bank Pharmaceuticals Inc.

\section{P01.02 HLA CLASS-I AND CLASS-II RESTRICTED NEOANTIGEN LOADS PREDICT OVERALL SURVIVAL IN BREAST CANCER}

${ }^{1} \mathrm{YW}$ Asmann*, ${ }^{1} \mathrm{Y}$ Ren, ${ }^{1} \mathrm{DP}$ Wickland, ${ }^{2} \mathrm{~V}$ Sarangi, ${ }^{2} \mathrm{~S}$ Tian, ${ }^{2} \mathrm{JM}$ Carter, ${ }^{2} \mathrm{AS}$ Mansfield, ${ }^{2}$ MS Block, ${ }^{1}$ ME Sherman, ${ }^{1} \mathrm{KL}$ Knutson, ${ }^{2} \mathrm{Y}$ Lin. ${ }^{1}$ Mayo Clinic, Jacksonville, $F L$, USA; ${ }^{2}$ Mayo Clinic, Rochester, MN, USA

\subsection{6/jitc-2020-ITOC7.15}

Background Tumors acquire numerous mutations during development and progression. These mutations give rise to neoantigens that can be recognized by $\mathrm{T}$ cells and generate antibodies. Tumor mutational burden (TMB) is correlated with, and has often been used as a surrogate of, neoantigen load, although that relationship is different depending on cancer types. Recent studies reported correlations between higher $\mathrm{TMB}$ and better overall survival after immune checkpoint blockade therapies in bladder, colorectal, head and neck, and lung cancers but not in breast cancer. On the other hand, the relationship between neoantigen load and survival has been controversial in literature. Higher neoantigen load has been linked to better overall survival in ovarian cancer and melanoma, but worse survival in multiple myeloma. Recently, no clear associations were found between neoantigen load and survival in 33 cancer types although only class-I restricted neoantigens were included.

Materials and Methods We developed a bioinformatics workflow, REAL-neo, for identification, quality control (QC), and prioritization of both class-I and class-II human leukocyte antigen (HLA) bound neoantigens that arise from tumor somatic single nucleotide mutations (SNM), small insertions and deletions (INDEL), and gene fusions. The correlations between TMB and neoantigen load per sample were calculated using Pearson Correlation Coefficient. TMB and neoantigen load comparisons between various groups were performed using Student's t-test. The survival analyses were performed using the Cox proportional hazards models while correcting for covariates.

Results We applied REAL-neo to 835 primary breast tumors in the Cancer Genome Atlas (TCGA) and performed comprehensive profiling and characterization of the predicted neoantigens. SNMs contributed to only $6.25 \%$ of the total neoantigens (\# of class-I vs. class-II neoantigens = 1: 3.5 ); INDELs accounted for $57.17 \%$ of the total (class-I : class-II= $1: 2$ ), and gene fusions were responsible for $36.58 \%$ of the total (class-I : class-II $=1: 2 \cdot 2$ ). TMB were positively correlated with total and each sub-categories of neoantigen load (class I: SNM: $\mathrm{r}=0.59, \mathrm{p}<2.2 \mathrm{E}-16$; INDEL: $\mathrm{r}=0.28, \mathrm{p}$ $<2.2 \mathrm{E}-16$; gene fusion: $\mathrm{r}=0.26, \mathrm{p}=2.01 \mathrm{E}-11$; class II: SNM: $r=0.47, p<2.2 \mathrm{E}-16$; INDEL: $r=0.16, p=1.7 \mathrm{E}-$ 05 ; gene fusion: $\mathrm{r}=0.31, \mathrm{p}=4.37 \mathrm{E}-13)$. The vast majority $(99.75 \%)$ of the predicted neoantigens occurred in $\leq 1 \%$ of the cases and $83.76 \%$ were patient-specific found in one patient only. Tumors with somatic and germline functional mutations in BRCA1 or BRCA2 genes had higher TMB ( $\mathrm{p}=$ 2.76E-06) and overall neoantigen load ( $p=0.009)$. Lower HLA class-I and class-II restricted neoantigen loads from SNM and INDEL were found to predict worse overall survival independent of TMB, breast cancer subtypes, tumor infiltrating lymphocyte (TIL) levels, tumor stage, and age at diagnosis (class-I: HR $=1.81, \mathrm{p}=0.04 ;$ class-II: HR $=1.89, \mathrm{p}=$ 0.042).

Conclusions Our study highlighted the importance of accurate and comprehensive neoantigen profiling and QC, and is the first to report the predictive value of neoantigen load for overall survival in breast cancer. This work was support by the State of Florida Cancer Center Grant, the bioinformatics program of Mayo Clinic Center for Individualized Medicine, and the Mayo Clinic inter-SPORE development grant.

Disclosure Information Y.W. Asmann: None. Y. Ren: None. D. P. Wickland: None. V. Sarangi: None. S. Tian: None. J.M. Carter: None. A.S. Mansfield: None. M.S. Block: None. M.E. Sherman: None. K.L. Knutson: None. Y. Lin: None. 